\title{
MULTI-GNSS RECEIVER FOR AEROSPACE NAVIGATION AND POSITIONING APPLICATIONS
}

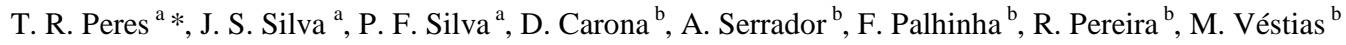 \\ a DEIMOS Engenharia, Av. D. João II, Lt 1.17.01, 10 Andar, 1998-023 Lisboa, Portugal - (joao.silva, tiago.peres, \\ pedro.silva)@deimos.com.pt \\ ${ }^{\mathrm{b}}$ Dept. of Electronics, Telecommunications and Computer Engineering, ISEL - Instituto Superior de Engenharia de \\ Lisboa, R. Conselheiro Emídio Navarro, 1, 1959-007 Lisboa, Portugal - (dcarona, aserrador)@ deetc.isel.pt, (a37579, \\ a32898)@alunos.isel.pt, mvestias@ deetc.isel.ipl.pt
}

KEY WORDS: Aerial, Land, Navigation, Georeferencing, GPS/INS, Interoperability, Hardware, Systems

\begin{abstract}
:
The upcoming Galileo system opens a wide range of new opportunities in the Global Navigation Satellite System (GNSS) market. However, the characteristics of the future GNSS signals require the development of new GNSS receivers. In the frame of the REAGE project, DEIMOS and ISEL have developed a GNSS receiver targeted for aerospace applications, supporting current and future GPS L1 and Galileo E1 signals, based on commercial (or, in the furthest extent, industrial) grade components. Although the REAGE project aimed at space applications, the REAGE receiver is also applicable to many terrestrial applications (ground or airborne), such as Georeferencing and Unmanned Aerial Vehicle (UAV) navigation. This paper presents the architecture and features of the REAGE receiver, as well as some results of the validation campaign with GPS L1 and Galileo E1 signals.
\end{abstract}

\section{INTRODUCTION}

The Galileo system (Europe's global navigation system, currently being deployed) and GPS modernization will soon provide new signals (with increasingly complex modulations and multiplexing schemes) which will enable performance enhancements in terms of availability, accuracy, and interference robustness of GNSS measurements and positioning solutions.

Galileo's E1 signal (European Union, 2010) and GPS's modernized L1 signal (GPS Directorate, 2011b), with their Multiplexed Binary Offset Carrier (MBOC) modulations, while not competing with Galileo's E5 signal (one of the most advanced and promising signals of the Galileo system), still provide a huge improvement in terms of precision and multipath robustness when compared with the current GPS L1 C/A and L2 C signals (GPS Directorate, 2011a), while keeping the required receiver complexity at a considerably low level.

Galileo's Initial Operational Capability (IOC) is expected by mid-decade (18 satellites, with which early services to Europe can begin (ESA, 2013a)) and Final Operational Capability (FOC) by 2020 (30 satellites, including 3 spares (ESA, 2013b)), while GPS modernization is ongoing and expected no sooner than 2025. With these parallel developments in mind and as a result of Europe's and USA's efforts towards Galileo-GPS interoperability, the future Galileo E1 and GPS L1 C signals will be compatible (Avila-Rodriguez, J. et al., 2007), enabling receiver simplification and increased availability at a low cost.

In the scope of the REAGE project (funded by ADI, under the Portuguese QREN initiative, contract no. 21553), DEIMOS and ISEL have developed a low-cost experimental multi-system
(Galileo and GPS) GNSS receiver for the L1/E1 band, targeted for use as a non-critical redundant sensor for space missions, but which can also be used for terrestrial (ground or airborne) navigation and positioning applications, such as Georeferencing and Unmanned Aerial Vehicle (UAV) navigation.

The REAGE project included the design and implementation of both RF front-end and baseband processor of a GPS/Galileo L1/E1 receiver, keeping it as flexible as possible to allow tradeoff analysis of receiver parameters and performances. The main purpose of this paper is to provide an overview of the REAGE receiver's architecture and functionalities.

Furthermore, the precision of a GNSS receiver's measurements depends, not only on the signal characteristics and DSP algorithms performance, but also on RF front-end characteristics, as overall noise figure, filter(s) bandwidth(s), ADC sampling frequency and local oscillator stability. Thus, this paper also addresses the impact of the RF front-end configuration on the quality of the GNSS receiver measurements.

Finally, results from validation tests in the GATE facility (a Galileo receiver test-bed based on Galileo pseudolites transmitting representative Galileo signals) are also presented in this paper, demonstrating the REAGE receiver's applicability.

\section{THE REAGE RECEIVER PROTOTYPE}

The REAGE project's primary goal was to build a space receiver for Low Earth Orbit (LEO) missions using available commercial grade (or, in the farthest extent, industrial grade) components. The selection criteria for the components and

\footnotetext{
* Corresponding author.
} 


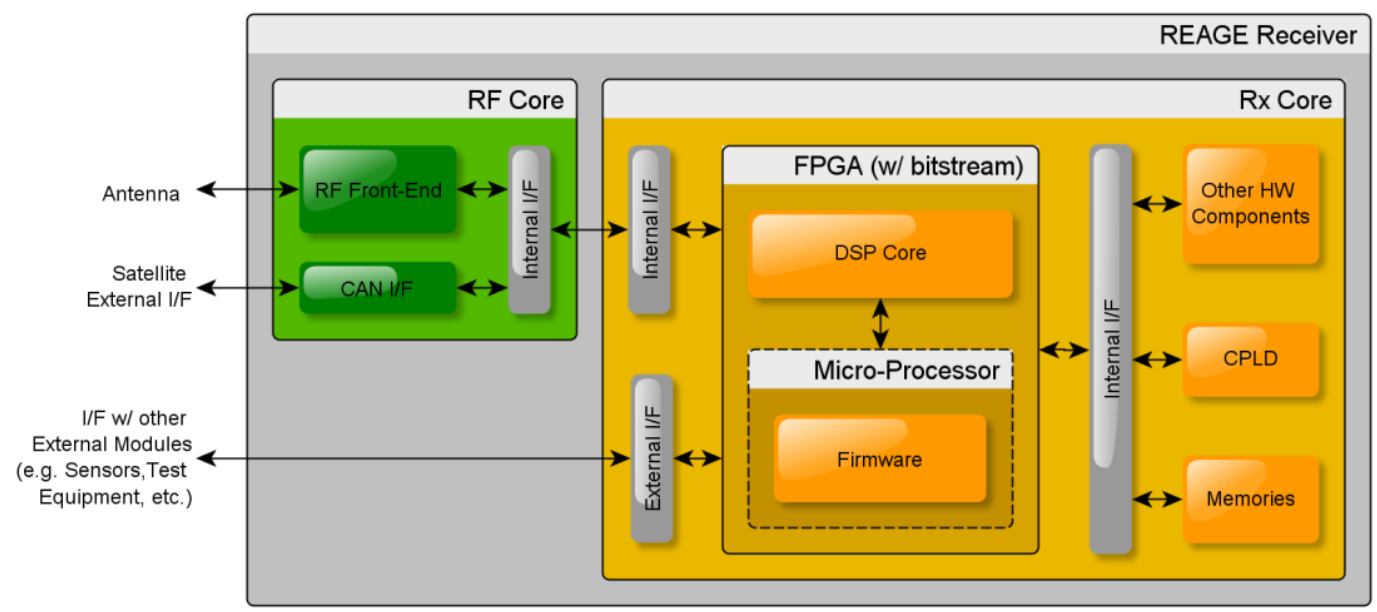

Figure 1. REAGE receiver architecture.

materials that compose each block of the receiver was based on an expected LEO mission duration of 7 to 10 years, which determines, for instance, the critical temperature constraints to guarantee operability, and also the fact that the receiver may accept gradual ionization energy deposit that eventually will lead to inoperability of the receiver at predicted End-Of-Life (EOL) stages. Space HW related features, as redundancy and remote re-configuration, were taken into account in the definition of the REAGE receiver architecture and may also be useful for terrestrial applications.

The REAGE receiver, whose architecture is illustrated in Figure 1 , is composed of two main modules:

- The RF Core, which includes the HW in charge of signal reception, conditioning, down-conversion, and sampling, as well as an external interface;

- The Rx Core, which includes a HW part, in charge of the high-frequency Digital Signal Processing (DSP) of the Intermediate Frequency (IF) and Baseband (BB) signals (implemented on a Field-Programmable GateArray (FPGA)), and a Software (SW) part, which controls the HW part of the Rx Core, generates measurements and computes the navigation solution.

The RF Core and Rx Core are further detailed in the following sections.

\section{RF Core}

The RF Core includes the receiver front-end and a Controller Area Network (CAN) interface, for communication with external systems (e.g. the satellite's on-board computer, external sensors or testing equipment).

The RF Core's front-end is based on a System-on-Chip (SoC) from Maxim IC, the MAX2769 (Maxim Integrated, 2010), and was selected taking into account the REAGE receiver's requirements. This chip has a complete receiver chain, including:

- Dual-Input Low-Noise Amplifier (LNA), supporting active and passive antennas;

- A Mixer, in charge of the down-conversion of the RF signal to baseband or low Intermediate Frequency (IF);

- A programmable Baseband/IF Filter, to select the signal band and reject out-of band interference;
- A Programmable Gain Amplifier (PGA), to increase the range and flexibility of the amplifying stages;

- A multi-bit (up to 4 bits) Analog-to-Digital Converter (ADC), to digitize the received signal (which is then fed to the Baseband Processor);

- And a Frequency Synthesizer (consisting of a Crystal Oscillator, a VCO and a fractional-N Frequency Divider), to generate the correct clock frequencies for the Mixer and ADC.

Figure 2 shows the developed RF Core prototype, which includes the RF front-end, based on the MAX2769 chip, as well as a CAN interface. The RF front-end includes two antenna inputs (one for active antennas and another for passive antennas) as well as a digital interface to use with external processing units (used to program the front-end as well as to send the digitized data to the base-band processor).

Figure 3 shows the block diagram of the RF Core prototype.

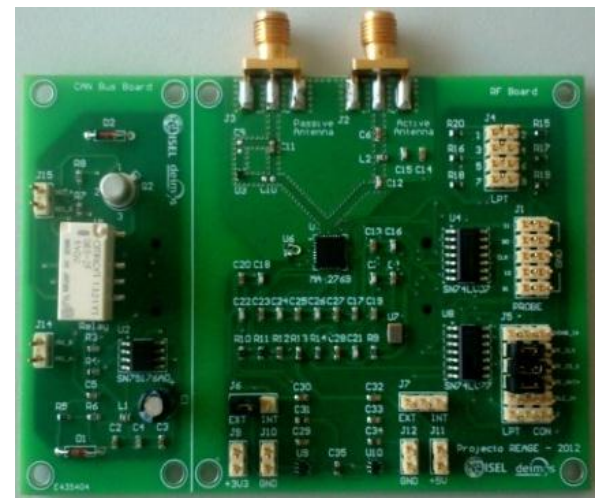

Figure 2. REAGE RF Core prototype (CAN interface on the left and RF front-end on the right).

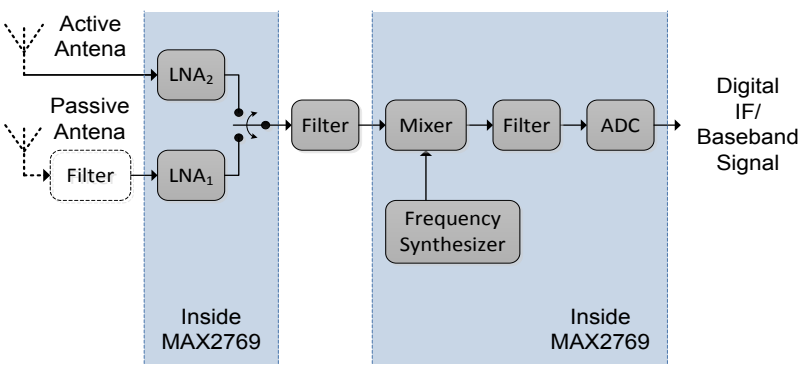

Figure 3. Developed RF front-end prototype block diagram. 


\section{Rx Core}

The baseband processor (Rx Core in Figure 1) is responsible for processing the IF/baseband signals coming from the RF frontend, generating measurements, computing the navigation solution and of overall system control and communication with external modules. It includes:

- An FPGA (a Xilinx Vitex-6), in which a DSP HW accelerator core and the receiver's processor (which runs the receiver firmware) are implemented;

- Memory modules, including RAM, used by the microprocessor, and Programmable ROM, used to store the FPGA's bitstream, the receiver's firmware and nonvolatile data;

- A CPLD-based system controller (in charge of receiver reconfiguration and external communication via the CAN interface on the RF Core);

- Different internal and external interfaces, for communication between the different receiver modules and between the receiver and the satellite and/or other external modules. Available interfaces include the RF Core interface (which also allows access to the CAN interface), Ethernet, UART, FMC and PCIe connectors, and General Purposes Input/Output (GPIO) pins, among others,

Figure 4 presents the high-level system decomposition, architecture and interfaces of the signal processing cores implemented by the Rx Core Bitstream on the Rx Core FPGA. The interfaces between the different modules and the rest of the receiver control system are also shown.

The FPGA hosts a set of Digital Signal Processing (DSP) modules (for the processing of the baseband GNSS signal), control modules (as the microprocessor and the Interrupt Control Unit), and interface modules (PCI and Ethernet controllers), as well as a few other general-purpose peripherals connected to the microprocessor and the main memory by means of a system bus. The following can be highlighted:

- The Input Modules, where the incoming signals are converted to baseband, filtered and re-quantized;

- The Channels, which are responsible for the GNSS baseband signal processing: carrier and code dispreading and samples accumulation;

- The Time Base Generator (TBG) which generates the Measurement Epoch and PPS signal;

- The Signal Level Detector, that accumulates signal occurrences in histogram bins which are used to estimate the signal power;

- The Interrupt Control Unit (ICU), which is responsible for monitoring the Integration Epoch, Measurement Epoch and PPS signal and generate the respective hardware interrupts which are forwarded to the microprocessor;

- The MicroBlaze (MB), a soft micro-processor on which the receiver firmware runs.

\section{Supported Signals and Outstanding Features}

The REAGE receiver supports current (legacy) and modernized GPS L1 civil signals (i.e. C/A and C) and Galileo E1 open service signals (i.e. B and C (European Union, 2010)).

Its Rx Core features programmable digital input filters, 16 independent and slavable HW channels (one "master" channel can be used to control other "slave" channels) - with code and carrier NCOs, mixers, primary and secondary code generators and 5 complex correlators each, for a total of 80 complex correlators -, and an embedded microprocessor (in charge of overall receiver control, acquisition, tracking loop closure, measurement generation, and navigation solution computation). The slavable and flexible GNSS channel design allows, for example, both pilot and data channels (when available, as is the case for GPS L1 C and Galileo E1 signals) to be processed coherently, or the extension of the delay line used to process each (or a particular) satellite (i.e. assigning more than 5 correlators to a satellite).

Furthermore, the REAGE receiver includes redundancy for some critical components and the ability of self-programming (in case the FPGA's bitstream becomes corrupted - due to radiation or any other reason - or if remote reconfiguration and/or updates are required).

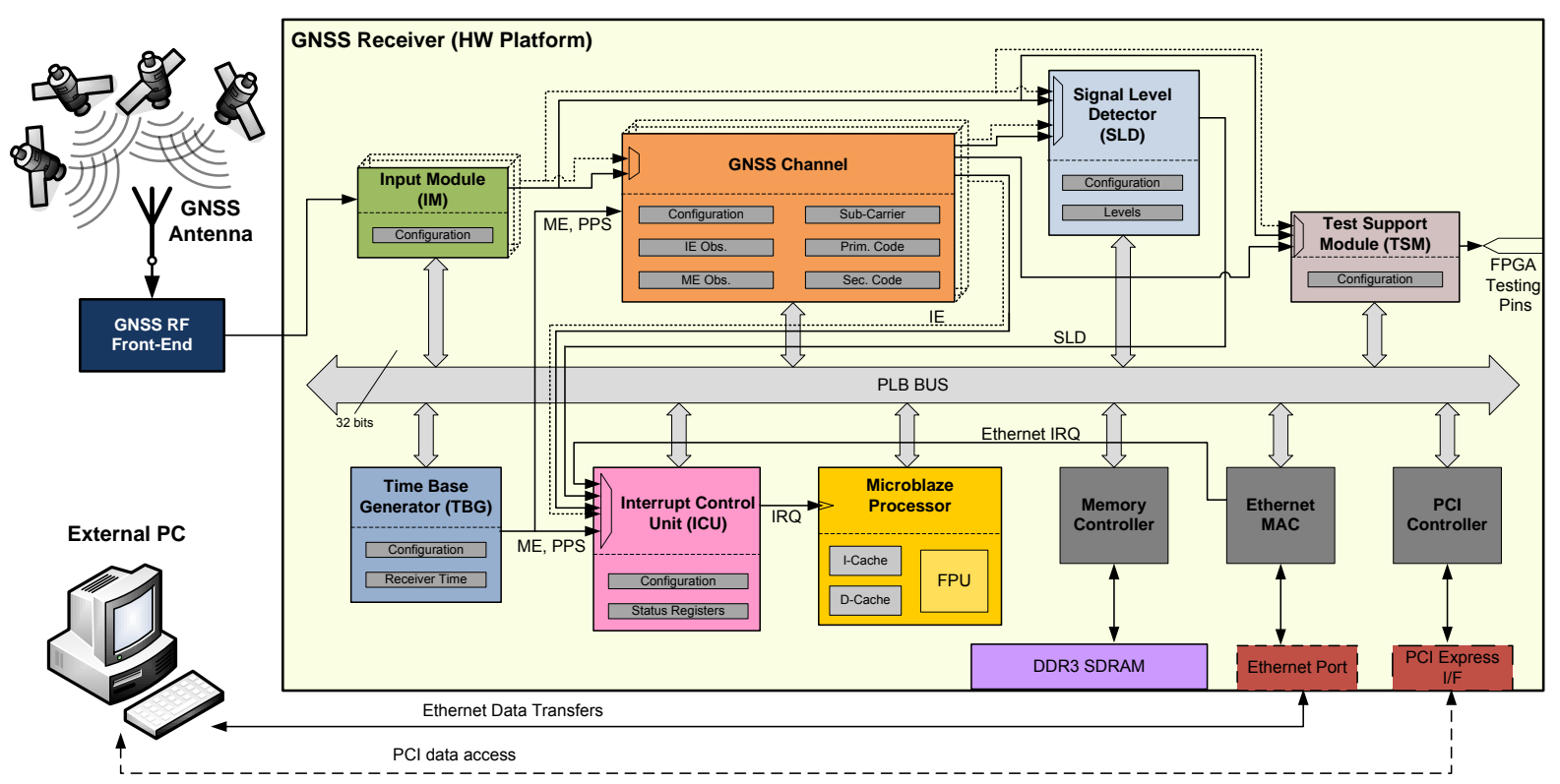

Figure 4. High-level architecture and interfaces of the Rx Core bitstream. 


\section{TEST RESULTS}

The REAGE receiver was tested in both static and dynamic (airborne) scenarios and for GPS L1 C/A and Galileo E1 signals. The aim of the tests was to do a preliminary assessment of the precision of the receiver's measurements as well as of its positioning solution for these scenarios.

Due to the currently limited visibility of Galileo satellites, the positioning performance tests with Galileo signals were done at the Galileo Test Range (GATE). The GATE test-bed features eight ground transmitters (pseudolites), emitting Galileo signals (fully compliant to the Galileo OS ICD specification, apart from minor differences in the navigation message contents) into the test area in the region of Berchtesgaden (in Germany).

At the time of writing, flight tests with the REAGE receiver onboard a UAV were not yet possible, therefore IF signals were recorded and later fed to the receiver for processing. GPS L1 and Galileo E1 signals were collected during the flight tests of the Portuguese Air Force's Alfa Extended UAV.

Two sets of test results are presented in this paper: results obtained in laboratory using GPS L1 C/A signals, for overall REAGE receiver validation and assessment of code tracking precision, and results obtained using GPS L1 C/A and Galileo E1 B signals collected at the GATE test-bed during flight tests.

\section{Code Tracking Performance}

The REAGE receiver was used to process GPS L1 C/A signals collected with a roof antenna at DEIMOS Engenharia's premises. For the purpose of performance comparison, three different RF front-end configurations were defined: a low bandwidth configuration $(2.5 \mathrm{MHz}$, the default configuration for the MAX2769 RF Front-end), a higher bandwidth configuration (8 MHz), and an intermediate bandwidth configuration (4.2 MHz). An IF frequency of $4 \mathrm{MHz}$ and a sampling frequency of $16.368 \mathrm{MHz}$ (default values for the MAX2769) were used for all tests. The correlator spacing was set to 1 sample (equivalent to 0.0625 chip for GPS L1 and Galileo E1 signals).

Figure 5 and Figure 6 show the (theoretical) Auto-Correlation Functions (ACFs) for the different bandwidths and signals. It can be seen that decreasing the bandwidth, besides implicating a loss of correlation power (more evident for the Galileo E1 signal due to its higher bandwidth), causes a rounding of the ACF peak. This reduces the receiver's tracking loops sensitivity, translating into higher measurement noise. Conversely, higher bandwidths, besides reducing correlation power losses due to filtering, enable a clearer discrimination of the ACF peak, decreasing measurement noise.

Figure 7 illustrates the distribution of the obtained code tracking errors for GPS L1 C/A for the different bandwidths (maximum, minimum, mean and quartiles, red circle is an identified outlier and was ignored). As expected, higher bandwidths are associated with lower code measurement noise (on average). It can be seen that the average code tracking noise drops from $3.3 \mathrm{~m}$, for $2.5 \mathrm{MHz}$ bandwidth, to $2.1 \mathrm{~m}$, for 4.2 MHz, and $1.75 \mathrm{~m}$, for $8 \mathrm{MHz}$. It can also be seen in Figure 7 that the improvement from increasing the front-end bandwidth from $4.2 \mathrm{MHz}$ to $8 \mathrm{MHz}$ is less evident than when the bandwidth changes from $2.5 \mathrm{MHz}$ to $4.2 \mathrm{MHz}$. This is also expected since the bandwidth of the GPS L1 C/A signal is only around $2 \mathrm{MHz}$. This could also be expected from the analysis of
Figure 6, which shows that the shape of the ACF is similar for both $4.2 \mathrm{MHz}$ and $8 \mathrm{MHz}$ of bandwidth. However, for the case of GPS L1 C or Galileo E1 signals, whose bandwidth is around $16 \mathrm{MHz}$ (although most of the power is concentrated in a bandwidth of $4 \mathrm{MHz}$ ), the improvement is expected to be more evident than for GPS L1 C/A signals, which is also suggested by Figure 6.

Based on the results presented above and on the Galileo E1 signal characteristics (CBOC $(6,1,1 / 11)$ modulation), it was expected that Galileo performances, in terms of code measurements noise, would outperform the ones for GPS L1 C/A by a factor of at least 3, as confirmed in other studies as the ENCORE project (Silva, P, et al., 2011), where measurement noise as low as $13 \mathrm{~cm}$ has been reported.

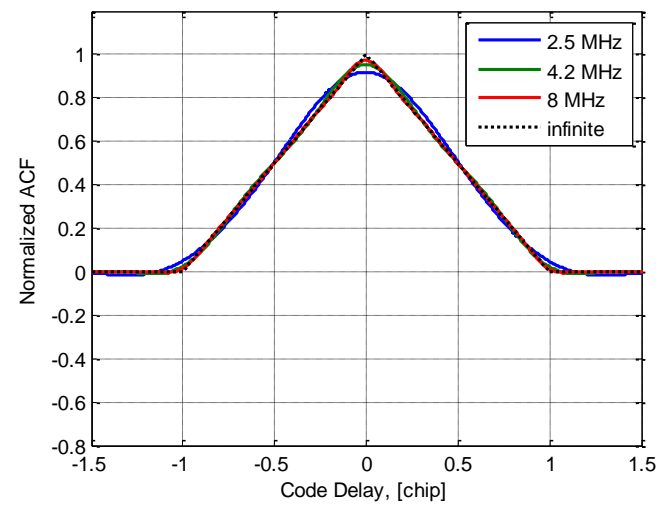

Figure 5. ACF of GPS L1 C/A signal for different RF front-end bandwidths.

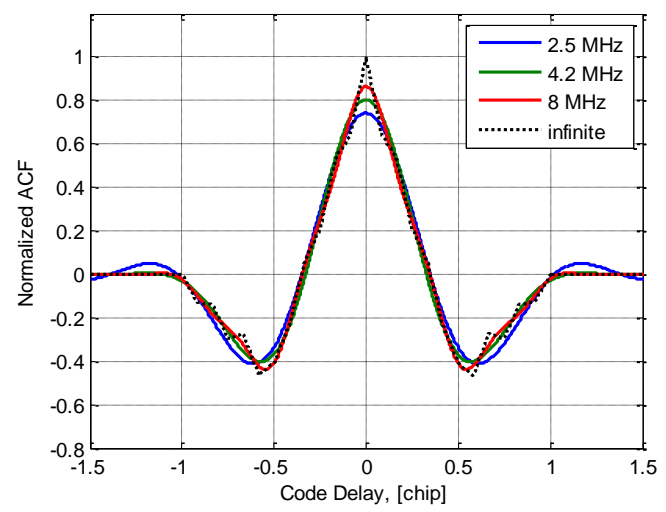

Figure 6. ACF of Galileo E1 B signal for different RF front-end bandwidths.

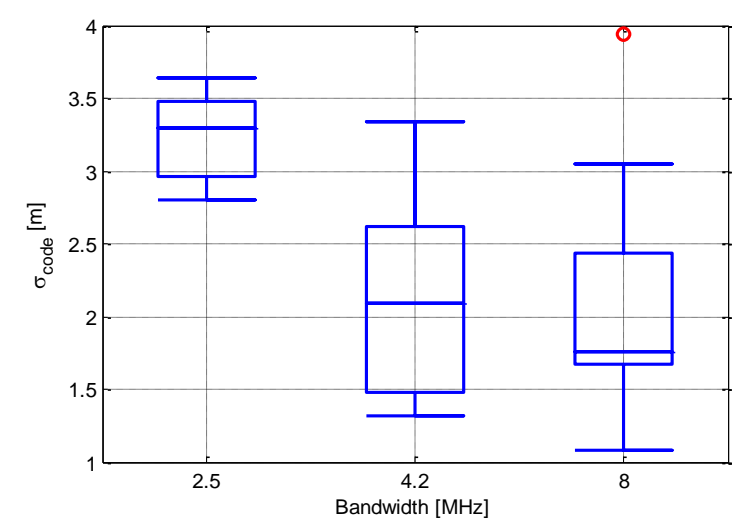

Figure 7. Distribution of code tracking errors for different bandwidths (for GPS L1 C/A signals). 


\section{Flight Trials}

Due to the low elevation of the Galileo pseudolites and the dynamics of the airborne scenario (frequent attitude changes which resulted in pseudolite masking), various losses of lock prevented an acceptable positioning solution from being reached for Galileo E1 signals. Therefore, positioning performance assessment with Galileo E1 signals was not possible. Nevertheless, real Galileo E1 signals were tracked and positioning results were attainable for GPS.

Table 1 summarizes the RF Front-End and receiver configurations used in the flight trials. A serial search algorithm was used for acquisition, while for tracking a $2^{\text {nd }}$ order PLL (for the carrier) and a $2^{\text {nd }}$ order PLL-aided DLL (for the code) were used. Figure 8 shows the UAV and the team involved in the flight trials at the test site. Figure 9 shows the visible GPS and Galileo satellites at the time of the test.

\begin{tabular}{|c|c|}
\hline Parameters & Values \\
\hline \multicolumn{2}{|c|}{ RF Front End Configuration } \\
\hline IF Sampling Frequency [Mhz] & 16.367667 \\
\hline IF $[\mathrm{MHz}]$ & 4.123968 \\
\hline IF Bandwidth [Mhz] & 4 \\
\hline \multicolumn{2}{|c|}{ Receiver Configuration } \\
\hline Integration Period [msec] & 4 \\
\hline Correlator Spacing $\left[\mathrm{T}_{\text {chip }}\right]$ & 0.13 \\
\hline Code Phase Discriminator & E-L Power \\
\hline DLL Bandwidth [Hz] & 2 \\
\hline Carrier Phase Discriminator & $\mathrm{Q} / \mathrm{I}$ \\
\hline PLL Bandwidth [Hz] & 12 \\
\hline
\end{tabular}

Table 1. RF Front-End and Receiver Configuration

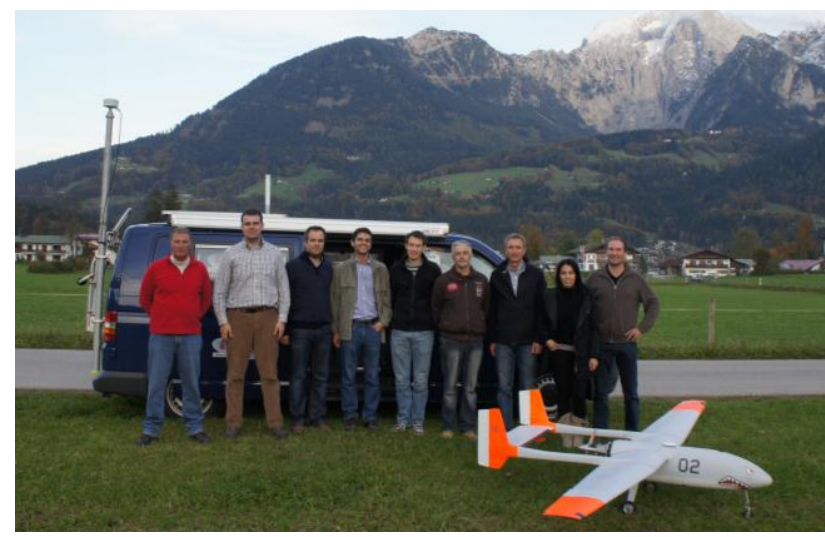

Figure 8. UAV and team involved in the flight trials.

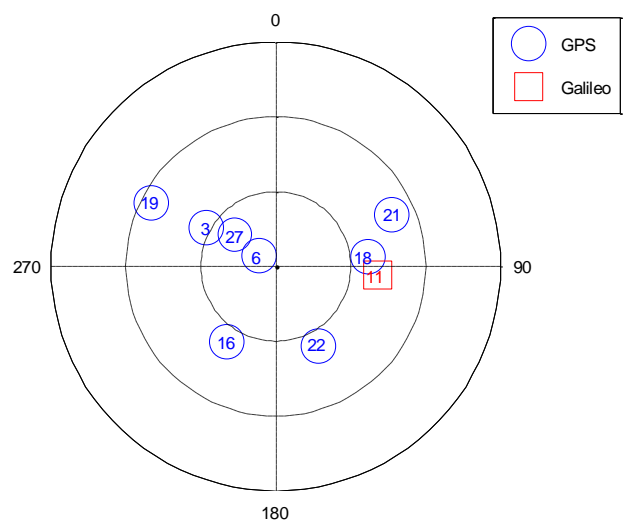

Figure 9. Galileo and GPS sky plot
Figure 10 shows the estimated Doppler for the signals which were successfully acquired and tracked by the receiver during the flight trial. The following should be noted:

- Before take-off, the receiver performed an initial search for all visible satellites. Since L1 SVID 21 was not found in the initial search (nearby trees may have obstructed visibility), this satellite was excluded from the acquisition search grid during the flight.

- During preparation for take-off, the GNSS antenna was obstructed several times and, because of this, some signals needed to be re-acquired (this was the case of L1 SVID 6 which was re-acquired after take-off).

- For L1 SVID 16 and E1 SVID 11 signals, tracking was lost during flight. This was probably due to the UAV manoeuvres (roll), which may have caused the signal to be obstructed by the wings.

- The UAV's dynamics resulted in Doppler variations with amplitudes up to $200 \mathrm{~Hz}$ in about 10 seconds for the case of E1 SVID 11 (see Figure 11). Simultaneously, there are also variations in the estimated $C / N_{0}$, probably due to both dynamic stress (affecting the quality of $C / N_{0}$ estimation) and antenna gain variations due to changes in the UAV roll angle.

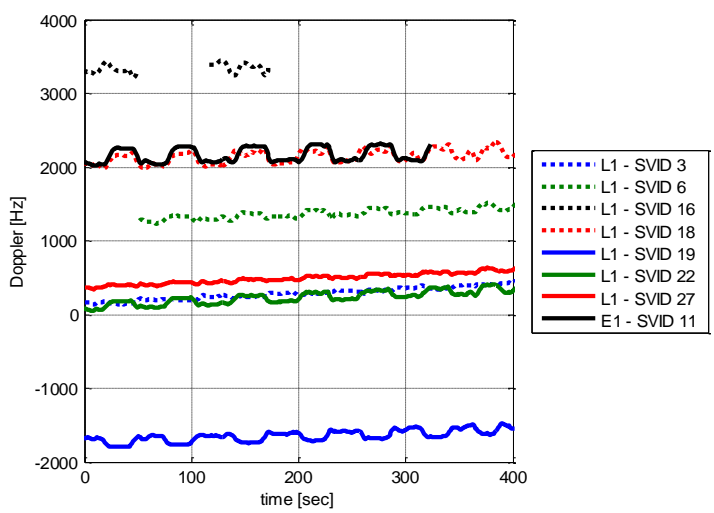

Figure 10. Estimated Doppler value for all tracked signals.
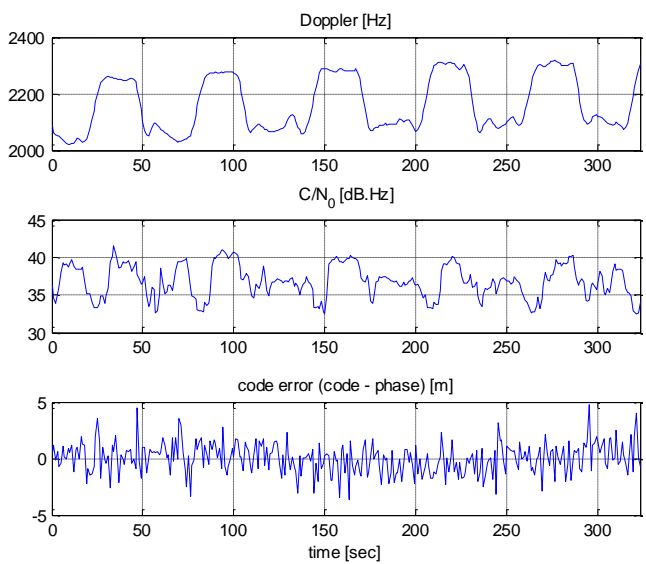

Figure 11. Doppler, $\mathrm{C} / \mathrm{N}_{0}$ and code error for E1 SVID 11.

Table 2 shows the elevation angle, estimated $C / N_{0}$ and estimated code error (computed as the difference between the code and carrier phase measurements) for all visible satellites. Figure 12 shows the estimated code error versus the estimated $C / N_{0}$. As expected, the higher the $C / N_{0}$, the lower the code error. The code error for the Galileo signal is also lower than the code error for GPS signals (with similar elevation angles and $C / N_{0}$ ). However, the observed performance improvement when using 
Galileo E1 was not as high as initially expected (the code error for E1 SVID 11 is less than $20 \%$ better than for L1 SVID 16), which could be explained by:

- The low pre-correlation bandwidth (4 MHz), which does not enable the full potential of E1 signals;

- Modifications made to the receiver to support a dynamic environment (including larger PLL bandwidth, use of a $2^{\text {nd }}$ order DLL, and different correlator spacings), which may not be optimized for Galileo E1 signals (further tuning will be made in future tests).

The position solution obtained for a period of the flight test (several figure-eights) is shown in Figure 11.

\begin{tabular}{|c|c|c|c|}
\hline Satellite & $\begin{array}{c}\text { Elevation } \\
{[\mathbf{d e g}]}\end{array}$ & $\begin{array}{c}\mathbf{C} / \mathbf{N}_{\mathbf{0}} \\
{[\mathbf{d B ~ H z}]}\end{array}$ & $\begin{array}{c}\text { Code Error } \\
{[\mathbf{m}]}\end{array}$ \\
\hline L1 - SVID 3 & 58 & 41.30 & 0.9364 \\
\hline L1 - SVID 6 & 82 & 40.19 & 1.0681 \\
\hline L1 - SVID 16 & 54 & 36.67 & 1.6674 \\
\hline L1 - SVID 18 & 53 & 38.28 & 1.3025 \\
\hline L1 - SVID 19 & 34 & 39.70 & 1.1060 \\
\hline L1 - SVID 22 & 54 & 40.33 & 1.0582 \\
\hline L1 - SVID 27 & 69 & 41.53 & 0.9214 \\
\hline E1 - SVID 11 & 49 & 36.85 & 1.3900 \\
\hline
\end{tabular}

Table 2. Elevation, $C / N_{0}$ and code error for all visible satellites

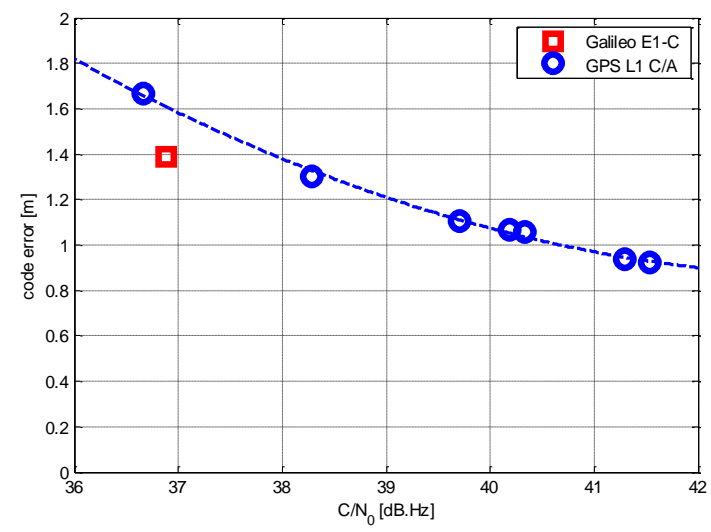

Figure 12. Estimated code error for all tracked signals

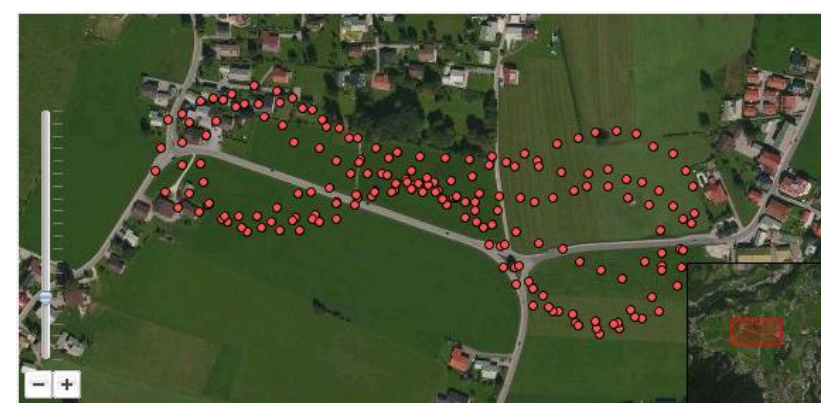

Figure 13. Estimated position during flight test

\section{CONCLUSIONS}

DEIMOS Engenharia and ISEL have developed a low-cost experimental multi-system (Galileo and GPS) GNSS receiver for the L1/E1 band, targeted for use as a non-critical redundant sensor for space missions but that can also be used for terrestrial applications (e.g. photogrammetry, UAV navigation), which relies on commercially available components.
This paper presented the architecture and main functionalities of the REAGE receiver, which supports current and future GPS L1 and Galileo E1 signals and whose flexibility makes it also useful for R\&D activities.

Experimental results from a test campaign which included the processing of GPS L1 C/A and Galileo E1 signals in static and dynamic (airborne) scenarios, were also presented, demonstrating the applicability of the receiver and providing preliminary performance figures.

\section{REFERENCES}

Avila-Rodriguez, J. et al., 2007. The MBOC Modulation - A Final Touch for the Galileo Frequency and Signal Plan. Inside GNSS, September/October 2007, Vol. 2, No. 6, pp. 43-58.

European Union, 2010. European GNSS (Galileo) Open Service Signal In Space Interface Control Document, OS SIS ICD, Issue 1.1, September 2010.

ESA, 2013a. "What is Galileo?", http://www.esa.int/Our_Acti vities/Navigation/The_future_-_Galileo/What_is_Galileo (14 Jan. 2013).

ESA, 2013b. Fact Sheet "Galileo Full Operational Capability Procurement", http://download.esa.int/docs/Galileo_IOV_Lau nch/FOC_factsheet_20111003.pdf (15 Jan. 2013).

GPS Directorate, 2011a. Navstar GPS Space Segment/ Navigation User Segment Interfaces, IS-GPS-200F, 21 Sep. 2011.

GPS Directorate, 2011b. Navstar GPS Space Segment/User Segment L1C Interfaces, IS-GPS-800B, 21 Sep. 2011.

GPS.Gov, 2013. "GPS Modernization”, http://www.gps.gov/sys tems/gps/modernization/ (15 Nov 2013)

Maxim Integrated, 2010. Datasheet "MAX2769 - Universal GPS Receiver", Rev 2, 6/10. http://datasheets.maximintegrated. com/en/ds/MAX2769.pdf (15 Mar. 2012).

Silva, P, et al., 2011. ENCORE: Enhanced Galileo Code Receiver for Surveying Applications. Proceedings of the ION GNSS 2011, Portland, Oregon, United States of America, September 2011.

\section{ACKNOWLEDGEMENTS}

The REAGE project was funded by the Portuguese Innovation Agency (ADI) under the Portuguese QREN initiative (contract no. 21553) and built upon the developments of the ENCORE project (GSA contract no. 952708) and GRIP project (internal). The authors would like to thank the respective project teams for their valuable contribution and support, in particular to José Maria Palomo, Carlos Valle and Juan Peres, from DEIMOS Space. The authors would also like to thank the Portuguese Air Force for providing the opportunity of testing the REAGE receiver with data collected during flight tests with their Alfa Extended UAV. 